DRAFT VERSION SEPTEMBER 10, 2018

Preprint typeset using LTEX style emulateapj v. 12/16/11

\title{
ELECTROMAGNETIC EMISSION FROM BLITZARS AND ITS IMPACT ON NON-REPEATING FAST RADIO BURSTS
}

\author{
Elias R. Most ${ }^{1}$, Antonios NATHANAIL ${ }^{1}$, AND LuCiano REZZOLla ${ }^{1,2}$ \\ Draft version September 10, 2018
}

\begin{abstract}
It has been suggested that a non-repeating fast radio burst (FRB) represents the final signal of a magnetized neutron star collapsing to a black hole. In this model, a supramassive neutron star supported by rapid rotation, will collapse to a black hole several thousand to million years after its birth as a result of spin down. The collapse violently snaps the magnetic-field lines anchored on the stellar surface, thus producing an electromagnetic pulse that will propagate outwards and accelerate electrons producing a massive radio burst, i.e., a "blitzar". We present a systematic study of the gravitational collapse of rotating and magnetised neutron stars with special attention to far-field evolution at late times after the collapse. By considering a series of neutron stars with rotation ranging from zero to millisecond periods and different magnetic-field strengths, we show that the blitzar emission is very robust and always characterised by a series sub-millisecond pulses decaying exponentially in amplitude. The luminosity and energy released when the magnetosphere is destroyed are well reproduced by a simple expression in terms of the stellar magnetic field and radius. Finally, we assess the occurrence of pair production during a blitzar scenario, concluding that for typical magnetic-field strengths of $10^{12} \mathrm{G}$ and spin frequencies of a few $\mathrm{Hz}$, pair production is suppressed. Overall, the very good match between the results of the simulations and the luminosities normally observed for FRBs lends credibility to the blitzar model as a simple and yet plausible explanation for the phenomenology of non-repeating FRBs.
\end{abstract}

Subject headings: black hole physics - MHD - methods: numerical - stars: neutron.

\section{INTRODUCTION}

The gravitational collapse of a magnetised and rotating neutron star can lead to interesting and multimessenger emission, both in terms of gravitational waves $(\mathrm{GW})$ and in terms of electromagnetic (EM) radiation in different bands. The detailed study of this combined emission can provide important insight into a number of astrophysical observations and, in particular, on a new type of astrophysical phenomena that is collectively referred to as fast radio bursts (FRBs, for a review Rane \& Lorimer (2017)).

FRBs are bright, millisecond radio single pulses that do not normally repeat and are not associated with a known pulsar or gamma-ray burst. The accounted dispersion measurements suggest that they are extragalactic, thus implying that their high radio luminosity is far larger than the single pulses from known pulsars. Furthermore, evidence of high magnetisation levels has been observed through Faraday rotation measurements close to the source of a single FRB 110523 (Masui et al. 2015) Because these transient radio sources are yet to be linked with confidence with a theoretical model, dozens of them exist in the literature, explaining either repeating FRBs such as Popov \& Postnov (2010); Pen \& Connor (2015); Lyubarsky (2014); Katz (2016); Cordes \& Wasserman (2016); Lyutikov et al. (2016) or FRBs that have been detected only once and that represent the large majority (Piro 2012; Totani 2013; Wang et al. 2016; Kashiyama et al. 2013; Mingarelli et al. 2015; Zhang 2016; Liebling \& Palenzuela 2016).

The "blitzar" model of Falcke \& Rezzolla (2014), is particularly relevant for our study as it involves the gravitational collapse of rotating and magnetised neutron stars. More specifically, in this model, an isolated and magnetised neutron star that was born massive enough, can be supported against

\footnotetext{
${ }^{1}$ Institut für Theoretische Physik, Goethe Universität Frankfurt, Maxvon-Laue-Str. 1, 60438 Frankfurt am Main, Germany

${ }^{2}$ Frankfurt Institute for Advanced Studies, Ruth-Moufang-Str. 1, 60438 Frankfurt am Main, Germany
}

gravitational collapse by its rapid rotation. However, the star is also continuously spinning down because of loss of kinetic rotational energy in the form of EM dipolar emission; the spindown continues up until the threshold to a dynamical instability to gravitational collapse - the neutral stability line - is reached and the star then collapses on a dynamical timescale (Takami et al. 2011; Weih et al. 2018). During the collapse, the magnetic field that was previously anchored on the surface of the star can either follow it as the surface is trapped behind the event horizon, or propagate outwards in the form of EM waves as the magnetic field lines are snapped. If the magnetic field is initially dipolar, the structure of these EM waves will be quadrupolar, with large magnetic blobs carrying away most of the EM energy. Furthermore, the travelling large-scale magnetic shock that propagate outwards can accelerate free electrons which will produce radio signals dissipating the radiated energy (Falcke \& Rezzolla 2014).

Together with EM radiation, the collapsing star will also produce $\mathrm{GW}$ radiation even if perfectly axisymmetric and simply because its quadrupole moment will have a nonzero time derivative. While we do not intend to focus here on the GW emission from these events, since detailed studies already exist [see, e.g., Baiotti et al. (2007) for a comprehensive discussion], we will rather concentrate on how the EM emission is produced during the collapse and how it propagates outwards as EM waves following the destruction of the largescale and ordered magnetosphere. In particular, our aim is to follow the evolution of the neutron star's magnetic field, both interior and exterior, during the collapse to a black hole and to quantify how the EM luminosity depends on the initial parameters of the neutron star, namely, its rotation rate and its magnetic-field strength.

We should recall that the collapse of a magnetised rotating neutron star is an old problem that has been studied rather extensively in the past, although only very recently in full general relativity. The first step was consid- 
ered by Wilson (1975), who simulated the collapse in an ideal-magnetohydrodynamical (MHD) framework including the magnetic field only in the interior of the star. While this work represented a first attempt to simulate this process, the fact that the magnetic field was isolated to the stellar interior had the consequence that no EM radiation was produced in the process, since the magnetic field is dragged with the collapsing matter and eventually hides behind a horizon. More recently, Baumgarte \& Shapiro (2003) investigated this scenario by considering the perturbative dynamics of the EM fields on a dynamical spacetime given by an Oppenheimer-Snyder collapse, that is, by the collapse of a nonrotating dust cloud. Starting with a dipolar magnetic field, Baumgarte \& Shapiro (2003) especially analysed how the magnetosphere exterior to the star changes during and mostly after the collapse of the star and the formation of the black hole. Furthermore, they showed that the magnetic flux decays exponentially in time after the collapse, following the quasi normal modes of the newly formed black hole, leaving an unstructured magnetic field in the vicinity of the black hole and outward propagating EM waves in the far field.

Much of this behaviour was later analysed and confirmed by Dionysopoulou et al. (2013), who studied similar initial conditions of a nonrotating neutron star, but where the spacetime was self-consistently evolved via the solution of the Einstein equations and the EM fields within a fully general-relativistic resistive-MHD framework. The gravitational collapse of two magnetised and rotating neutron stars was instead investigated by Lehner et al. (2012), who considered and contrasted two different and extreme magnetospheric conditions: electrovacuum and force-free. In particular, they showed that, in the force-free case, the magnetic flux threading the event horizon completely vanishes within a millisecond from black-hole formation, mostly because of reconnection. On the other hand, in the case of the electrovacuum simulation. it was shown that the EM emission depends weakly on rotation. Following the work of Dionysopoulou et al. (2013) in resistive MHD and extending it to the case of rotating magnetised neutron stars in electrovacuum, Nathanail et al. (2017) have recently shown that the initial charge of the neutron star can be trapped behind the apparent horizon, thus forming a charged black hole of Kerr-Newman type.

We here further explore the scenario investigated by Nathanail et al. (2017) focussing mostly on the late time evolution of the far field, where we can identify and analyse the millisecond-long EM pulses produced during the gravitational collapse and the violent disruption of the magnetosphere. Once produced in the vicinity of the surface of the star and the apparent horizon that soon forms, these EM pulses propagate outwards carrying enormous amounts of energy, with magnitudes which are in good agreement with that associated with FRBs. Furthermore, we here explore how the energetics of the emission depends on the basic properties of the stars, i.e., spin rate and magnetic-field strength, and provide a simplified algebraic expressions that reproduces well our results.

The plan of the paper is the following one. In Sec. 2 we briefly review the numerical setup and how the initial data is computed. In Sec. 3 we discuss the significance of our results due to pair creation during collapse. The analysis of the numerical results follows in Sec. 4. Finally, the discussion of the astrophysical impact of the results and our conclusions are presented in Sec. 5.

\section{NUMERICAL SETUP AND INITIAL DATA}

The simulations reported below have been performed using the general-relativistic resistive-MHD code WhiskyRMHD (Dionysopoulou et al. 2013, 2015). The code uses highresolution shock-capturing methods such as the Harten-Laxvan Leer-Einfeldt (HLLE) approximate Riemann solver. Following Nathanail et al. (2017), we reconstruct our primitive variables at the cell interfaces using the enhanced piecewise parabolic reconstruction (ePPM), which does not reduce to first order at local maxima (Colella \& Sekora 2008; Reisswig et al. 2013). For the evolution of the spacetime, the WhiskyRMHD code makes use of the Einstein Toolkit framework (Löffler et al. 2012), which exploits the McLachlan code for the space-time evolution and the Carpet driver for fixed-box mesh refinement (Schnetter et al. 2004). An important point in our resistive-MHD treatment is the calculation of the electric charge $q$, which we compute at every timestep via the divergence of the electric field, i.e., $q=\nabla_{i} E^{i}$, as adopted in several other works (Bucciantini \& Del Zanna 2013; Dionysopoulou et al. 2013; Qian et al. 2017; Nathanail et al. 2017).

Because the focus of our study is mainly the evolution of the magnetic field in the exterior of the star and the luminosity produced during and after the collapse, the use of a resistive-MHD framework is particularly convenient. In particular, we can assume a negligibly small electrical conductivity in the exterior of the star so that it can effectively (although not exactly) reproduce an electrovacuum regime. At the same time, we can use a very large value of the electrical conductivity in the stellar interior so that we can reproduce the highly conducting matter. However, connecting the two regimes of low and high conductivity across the star and its exterior has the consequence that the set of resistive-MHD equations becomes stiff and hence requires special time stepping strategies. Following Palenzuela et al. (2009), we employ an implicit-explicit Runge-Kutta time stepping (RKIMEX) algorithm (Pareschi \& Russo 2005), the details of our implementation can be found in Dionysopoulou et al. (2013) and Dionysopoulou et al. (2015). We use the same setup here as Nathanail et al. (2017) and choose a finest resolution of $147 \mathrm{~m}$ with a total domain size of $1075 \mathrm{~km}$, which in combination with the implicit time evolution scheme makes these runs rather expensive.

The initial neutron-star models are computed using the Magstar code (Bocquet et al. 1995) of the LORENE library (www. lorene.obspm.fr). In particular, Magstar computes self-consistent uniformly rotating neutron stars by solving the coupled system of the Einstein-Maxwell equations. In this way, and using a polytropic equation of state (Rezzolla \& Zanotti 2013) $p=K \rho^{\Gamma}$ with $\Gamma=2$ and $K=164.708$ we have computed a total of 17 initial stellar models, whose properties are collected in Table 1. The evolutions have been performed using a simple gamma-law $p=\rho \varepsilon(\Gamma-1)$ to allow for shock heating. Note that each model is characterised by a rotational frequency ${ }^{3}$ and by a magnetic-field strength ${ }^{4}$;

\footnotetext{
${ }^{3}$ Note that we choose an upper limit for the spinning frequency of $800 \mathrm{~Hz}$ because this is already higher than the fastest pulsar presently observed, i.e., PSR J1748-2446ad, whose rotation period is $1.3 \mathrm{~ms}$ (Hessels et al. 2006).

${ }^{4}$ While a magnetic field of $10^{13} \mathrm{G}$ is about two orders of magnitude larger than what is normally expected in a blitzar. As we will show in Sec. 4.2, the results follow a simple scaling relation with the magnetic-field strength, but using a large value reduces the computational costs.
} 


\begin{tabular}{|c|c|c|c|c|c|c|c|}
\hline & \begin{tabular}{|c}
$M$ \\
{$\left[M_{\odot}\right]$}
\end{tabular} & $\begin{array}{c}R_{c} \\
{[\mathrm{~km}]}\end{array}$ & $\begin{array}{r}f_{\text {spin }} \\
{[\mathrm{Hz}]}\end{array}$ & $\begin{array}{c}J / M^{2} \\
-\end{array}$ & $\begin{array}{c}B_{\mathrm{pol}} \\
{\left[10^{13} \mathrm{G}\right]}\end{array}$ & $\begin{array}{c}E_{\mathrm{EM}} \\
{\left[10^{43} \mathrm{erg}\right]}\end{array}$ & $\begin{array}{l}E \\
-\end{array}$ \\
\hline F000.B13 & 2.096 & 14.940 & 0 & 0.000 & 6.09 & 22.03 & zero \\
\hline . B12 & 2.076 & 15.546 & 1 & 0.0005 & 0.217 & $3.12 \mathrm{E}-2$ & corotating \\
\hline . B13 & 2.096 & 14.941 & 10 & 0.005 & 2.39 & 3.50 & GR rot. sphere \\
\hline . B13 & 2.097 & 14.945 & 50 & 0.025 & 2.29 & 3.06 & corotating \\
\hline B13 & 2.095 & 15.069 & 80 & 0.041 & 2.28 & 3.02 & corotating \\
\hline & 2.084 & 15.442 & 100 & 0.053 & 2.22 & 5.57 & GR rot. sphere \\
\hline F14 & 2.095 & 15.216 & 140 & 0.072 & 2.27 & 2.99 & corotating \\
\hline & 2.092 & 15.511 & 200 & 0.107 & 2.26 & 3.04 & corotating \\
\hline F3 & 2.081 & 16.190 & 300 & 0.174 & 2.23 & 4.43 & GR rot. sphere \\
\hline F 4 & 2.103 & 16.404 & 400 & 0.234 & 2.33 & 2.85 & corotating \\
\hline F4 & 2.132 & 16.069 & 450 & 0.251 & 2.44 & 3.61 & GR rot. sphere \\
\hline & 2.144 & 17.2 & 550 & 0.3 & 2.50 & 1.80 & corotating \\
\hline & 2.179 & 16.946 & 600 & 0.357 & 2.63 & 4.49 & GR rot. sphere \\
\hline F 8 & 2.104 & 9.909 & 800 & 0.314 & 52.84 & 0.87 & GR rot. sphere \\
\hline & 2.147 & 16.210 & 500 & 0.281 & 0.0025 & $2.37 \mathrm{E}-6$ & corotating \\
\hline F5 & 2.147 & 16.210 & 500 & 0.281 & 0.25 & $2.37 \mathrm{E}-2$ & ating \\
\hline & 2.147 & 16.210 & 500 & 0.281 & 25 & $2.68 \mathrm{E}+2$ & corotating \\
\hline & 2.147 & 16.210 & 500 & 0.281 & 250 & $4.13 \mathrm{E}+4$ & corotating \\
\hline
\end{tabular}

TABLE 1

INITIAL NEUTRON-STAR MODELS. REPORTED IN THE DIFFERENT COLUMNS ARE: THE ADM MASS $M$, THE CIRCUMFERENTIAL RADIUS $R_{C}$, THE SPIN FREQUENCY $f_{\text {spin }}$, THE DIMENSIONLESS ANGULAR MOMENTUM WITH $J$ THE KOMAR ANGULAR MOMENTUM, THE VALUE OF THE MAGNETIC FIELD AT THE POLE OF THE NEUTRON STAR $B_{\text {pol }}$, THE RADIATED EM ENERGY $E_{\text {EM }}$, AND THE TYPE OF INITIAL ELECTRIC-FIELD CONFIGURATION. NOTE THAT THE VALUE OF THE MAXIMUM MAGNETIC FIELD $B_{c}$ INSIDE THE NEUTRON STAR, IS A FACTOR OF $\sim 6$ LARGER IN ALL MODELS.

for instance, model $\mathrm{F} 300 . \mathrm{B} 13$ refers to a magnetised neutron star with spin frequency of $f=300 \mathrm{~Hz}$ and a dipolar magnetic field with a value at the pole $B_{\mathrm{pol}}=10^{13} \mathrm{G}$. Collectively, the models presented in Table 1 can be considered as representative of the magnetic field strengths and of the rotational frequencies to be expected by supramassive magnetised neutron stars just before collapse.

Although the solution provided by Magstar includes selfconsistent electric and magnetic fields, a certain freedom remains in the choice of an initial electric field that is consistent with our electrovacuum prescription. In fact, while the electric field in the neutron-star interior is always unambiguously given by the ideal-MHD condition i.e., $E^{i}=-\epsilon^{i j k}\left(v_{\mathrm{c}}\right)_{j} B_{k}$, where $\left(v_{\mathrm{c}}\right)_{j}$ is the corotation velocity and $\epsilon^{i j k}$ the totally antisymmetric permutation symbol, the electric field outside the star should be such that there are no charges outside, i.e., the electric field should be divergence free in the stellar exterior. A similar ambiguity was discussed by Nathanail et al. (2017), who, after considering several different options, found that the optimal initial electric field minimising the exterior charge density is the one deriving from the analytical solution of a rotating magnetised sphere in general relativity (Rezzolla et al. 2001, 2003).

Alternative approaches using a force-free description of the magnetosphere have instead prescribed the electric field in terms of the corotation velocity and of the magnetic field (Lehner et al. 2012; Palenzuela 2013); this approach is appropriate inside the light cylinder $r_{L}:=c / \Omega$ of such a magnetosphere.

Fortunately, the results of the simulations do not depend sensitively on the choice made for the electric field and, as we show in Appendix A, the variation of the EM luminosity with the different prescriptions is minimal and the light curves overlap over the whole duration of the intense EM emission. Hence we have opted for the most simple and robust prescription of prescribing the electric field using the corotation velocity as this does not require corrections for deviations from spherical symmetry in the case of the fast rotating models. Nonetheless, we have also performed some simulations with the rotating magnetised sphere prescription for several models to give an error range to our calculations as detailed in the
Appendix A.

\section{PAIR PRODUCTION}

Since we will be modelling the blitzar model in a purely electrovacuum scenario, it is important to check if this is a reasonable approximation and whether, instead, electronpositron pair production needs to be properly taken into account in this scenario. In view of this, in what follows we explore the significance of pair production during the collapse of a rotating neutron star. In essence, we review the basic mechanisms of pair production, as known from studies of pulsar magnetospheres (Harding \& Lai 2006), concentrating in particular on the photon-photon and photon-magnetic field mechanisms.

We recall that the occurence of photon-photon pair creation in a pulsar depends sensitively on two fundamental parameters: the surface temperature of the neutron star and the magnetic-field strength. Since the blitzar model involves supramassive neutron stars that are thousands to millions of years old, their surface temperature $T_{s}$ is expected to be well below $T_{s}<10^{6} K$ (Chabrier et al. 2006). For such temperatures in the pair formation region, the field strength should be $B>0.1 B_{\text {cr }}$ Harding \& Muslimov (2001), where $B_{\text {cr }}:=4.4 \times 10^{13} \mathrm{G}$ is the so-called critical magnetic-field strength. Thus, for an initial magnetic field of $\leq 4.4 \times 10^{12} \mathrm{G}$, normally expected in a blitzar and the one considered in our simulations here, photon-photon pair creation is strongly surpressed.

Another source of pair production is the photon-magneticfield mechaniscm, which involves the interaction of high energy photons with strong magnetic field, which proceeds as follows. When in the exterior of the pulsar a region is emptied of charges, thus creating a so-called "gap", an electric field parallel to the magnetic field develops as a result of unipolar induction. This huge voltage drop across magnetic field lines is capable of pulling charges from the neutron star surface and accelerate them to high Lorentz factors. These accelerated charges, radiate high-energy curvature photons, which, in turn, produce the electron-positron pairs through a cascade interaction with the magnetic field.

More specifically, the accelerated charges will attain a Lorentz factor $\gamma$ that is proportional to the developed voltage 
drop $\Delta V$ and is given by

$$
\gamma=e \Delta V / m_{e} c^{2}
$$

where $e$ and $m_{e}$ are the is the electric charge and the mass of the electron, respectively. The emitted radiation will have a characteristic frequency given by

$$
\nu_{c}=\gamma^{3} \frac{c}{r_{c}}
$$

where $r_{c}$ is the radius of curvature of the magnetic-field line that the charge will travel on. Electron-positron pairs are then created if (Sturrock 1971; Ruderman \& Sutherland 1975)

$$
\gamma^{3}\left(\frac{\hbar c}{2 m_{e} r_{c} c^{2}} \sin \theta\right)\left(\frac{B_{\mathrm{loc}}}{B_{\mathrm{cr}}}\right) \simeq \frac{1}{15},
$$

where $B_{\text {loc }}$ is the local magnetic field and $\sin (\theta)$ is the "pitch" angle between the photon and the magnetic-field line. It is common to write $\sin \theta \simeq h / r_{c}$, where $h$ is the length of the gap with the parallel electric field, (see, e.g., Chen \& Ruderman (1993)). Using now Eqs. (3) and (1), we find the criterion for triggering pair creation

$$
\begin{aligned}
\Delta V<\Delta V_{\mathrm{pp}} \simeq 3 \times 10^{15} & \left(\frac{r_{c}}{20 \mathrm{~km}}\right)^{2 / 3}\left(\frac{B_{\mathrm{loc}}}{10^{10} \mathrm{G}}\right)^{-1 / 3} \\
& \left(\frac{h}{0.2 \mathrm{~km}}\right)^{-1 / 3} \text { statV }
\end{aligned}
$$

or equivalently

$$
\begin{aligned}
E<E_{\mathrm{pp}} \simeq 1.5 \times 10^{11} & \left(\frac{r_{c}}{20 \mathrm{~km}}\right)^{2 / 3}\left(\frac{B_{\mathrm{loc}}}{10^{10} \mathrm{G}}\right)^{-1 / 3} \\
& \left(\frac{h}{0.2 \mathrm{~km}}\right)^{-4 / 3} \text { statV } / \mathrm{cm} .
\end{aligned}
$$

In other words, no pair creation is expected from the interaction of photons with the magnetic field as long as the voltage drop is below the critical one $\Delta V_{\mathrm{pp}} \sim 3 \times 10^{15} \mathrm{statV}$. For a typical pulsar, the voltage drop can be estimated as

$$
\begin{gathered}
\Delta V_{\text {typ }} \sim 1.2 \times 10^{13}\left(\frac{B_{\text {loc }}}{10^{12} \mathrm{G}}\right)\left(\frac{\Omega}{1880 \mathrm{rad} / \mathrm{s}}\right) \\
\left(\frac{h}{0.2 \mathrm{~km}}\right)^{2} \text { statV },
\end{gathered}
$$

where $\Omega$ is the angular velocity of the star.

While the estimate (6) is simple to carry out for a stationary pulsar, determining whether the voltage drop is always below the critical one in a collapsing scenario, where all quantities in Eq. (4) change dynamically, is obviously more complicated. In particular, during the collapse, the magnetic-field lines are changing rapidly and the path of the accelerated charge is not prescribed, but would need to be found self-consistently ${ }^{5}$. It follows that the curvature radius will also vary dynamically and any reference scale will not be valid but for a short time interval. Finally, within microseconds the magnetic field in the region within which the acceleration takes place may even change polarity, which means that the path that the charge will

\footnotetext{
5 Note that the light travel-time over a scale height of of $\sim 10 \mathrm{~km}$ is $\sim$ $30 \mu \mathrm{s}$, which is longer than the typical timescale of variation of the magneticfield lines.
}

follow might be chaotic, which questions the efficiency of the curvature process.

Notwithstanding these caveats, in Sec. 4.3 we will follow the evolution of the parallel electric field in order to check whether or not a sufficient voltage drop is created during the collapse and hence whether pair production is at work. We can already anticipate here that while pair production can take place during the collapse for sufficiently large magnetic fields, this process is not efficient for the typical values of the initial magnetic field in blitzars.

\section{NUMERICAL RESULTS}

In what follows we will present the results of the simulations involving the 17 neutron-star models that we have simulated. We recall that the bulk properties of the matter dynamics have already been studied in detail by several authors, starting from Font et al. (2002), and more recently by Baiotti et al. (2007) and Liebling et al. (2010). As shown in detail in those works, the hydrodynamical collapse to a black hole proceeds rapidly - essentially on a dynamical timescale - and does not leave any remnant matter outside the apparent horizon, thus fully justifying the assumption of an electrovacuum as the background over which the electromagnetic waves emitted during collapse will propagate in electrovacuum. Furthermore, since the overall phenomenological evolution is very similar for all of the models described in Table 1, we will discuss in more detail only the results for the models $\mathrm{F} 000 . \mathrm{B} 13, \mathrm{~F} 300 . \mathrm{B} 13$ and F600.B13 as they are three representative of three qualitatively different behaviours. More specifically, we will next first discuss the dynamics of the magnetic field during the collapse (Sec. 4.1) and subsequently the properties of the EM emission and the magnitudes of the energy losses (Sec. 4.2).

\subsection{Magnetic-field dynamics}

Although the nonrotating model F000.B13 was already considered by Dionysopoulou et al. (2013), we will briefly discuss it here as it provides a useful reference solution. We recall that the neutron star is initially endowed with a dipolar magnetic field, as can be seen in the first panel of Fig. 1. When the collapse begins, a strong discontinuity is produced in the magnetosphere as the whole surface of the star suddenly starts to move inwards. This "magnetic shock" propagates outwards at the speed of light, reaching the at $\sim 300 \mathrm{~km}$ in almost $\sim 1 \mathrm{~ms}$ and essentially destroys the dipolar field structure (see middle panel of Figs. 1,2). Behind this shock, and when the apparent horizon is formed, the magnetic-field lines are violently snapped. At this point, quadrupolar EM radiation is produced and the EM fields propagate outwards essentially as EM waves in electrovacuum. At the same time, the electric and magnetic fields near the stellar surface and the apparent horizon constantly decay, losing any ordered largescale structure as they cannot be sourced by the emerging Schwarzschild black hole (see right panel of Fig. 1). Figure 2 provides essentially the same information reported by Fig. 1, but it shows it on a larger scale of $\sim 450 \mathrm{~km}$ so as to highlight the coherent large-scale structure of the magnetic field and the quadrupolar nature of the emitted EM radiation.

What cannot be shown in detail in Figs. 2 and 1 are the magnetic-field properties near the apparent horizon. A careful analysis of the dynamics of the magnetic-field lines reveals that when the apparent horizon is formed, magneticfield lines still pass through it. However, as the black hole 

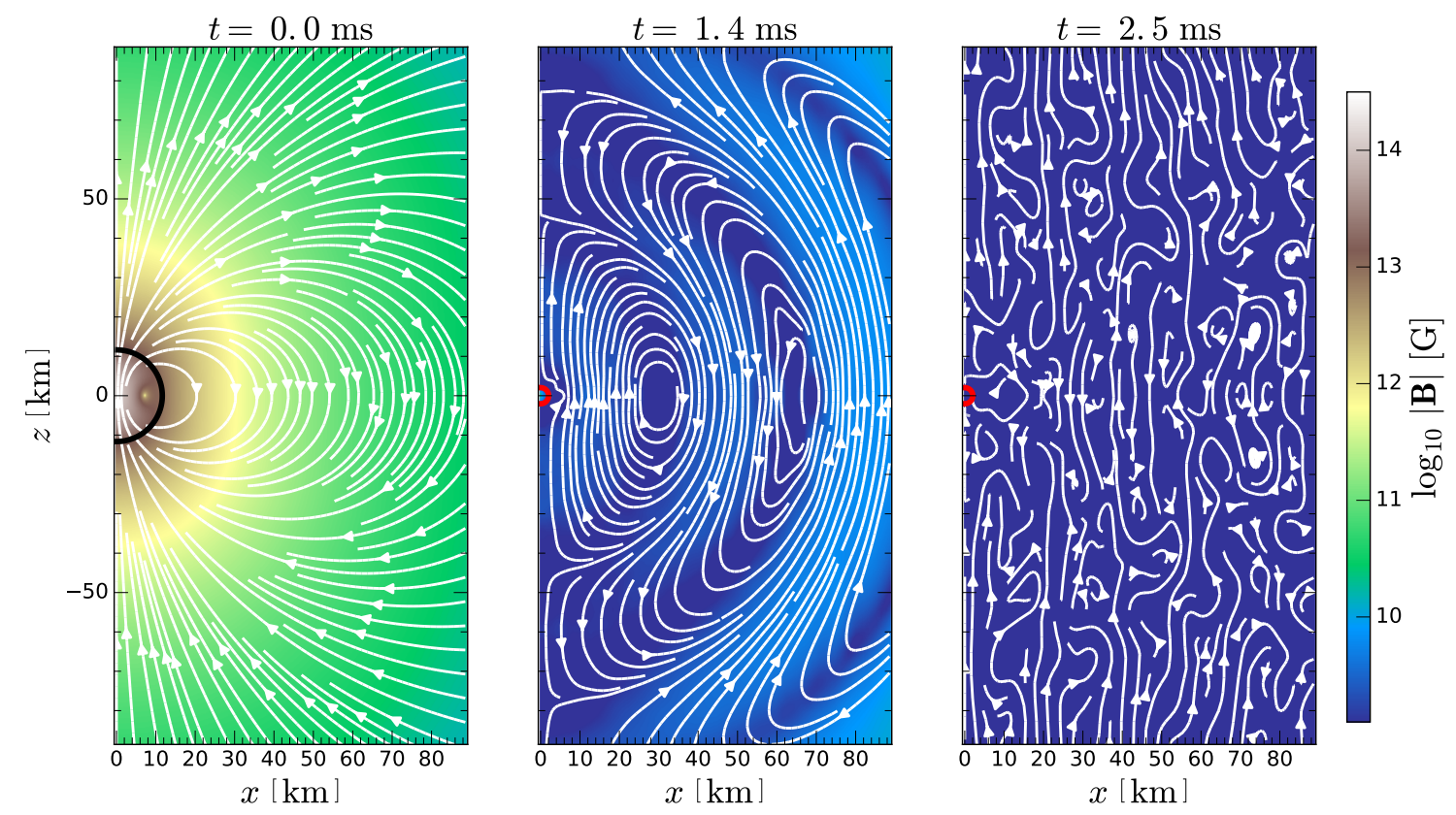

FIG. 1.- Magnetic field strength $|\boldsymbol{B}|$ in the $(x, z)$ plane shown with a colorbar and at three different times for the nonrotating model F000. B13. Also reported are the stellar surface (solid black line in the left panel), the apparent horizon (solid red line in the middle and right panels), and the magnetic-field lines (white lines). The initial magnetic field strength at the pole is $10^{13} \mathrm{G}$; note the lack of a final ordered magnetic field at late times (right panel) since the black hole produced is of Schwarzschild type.
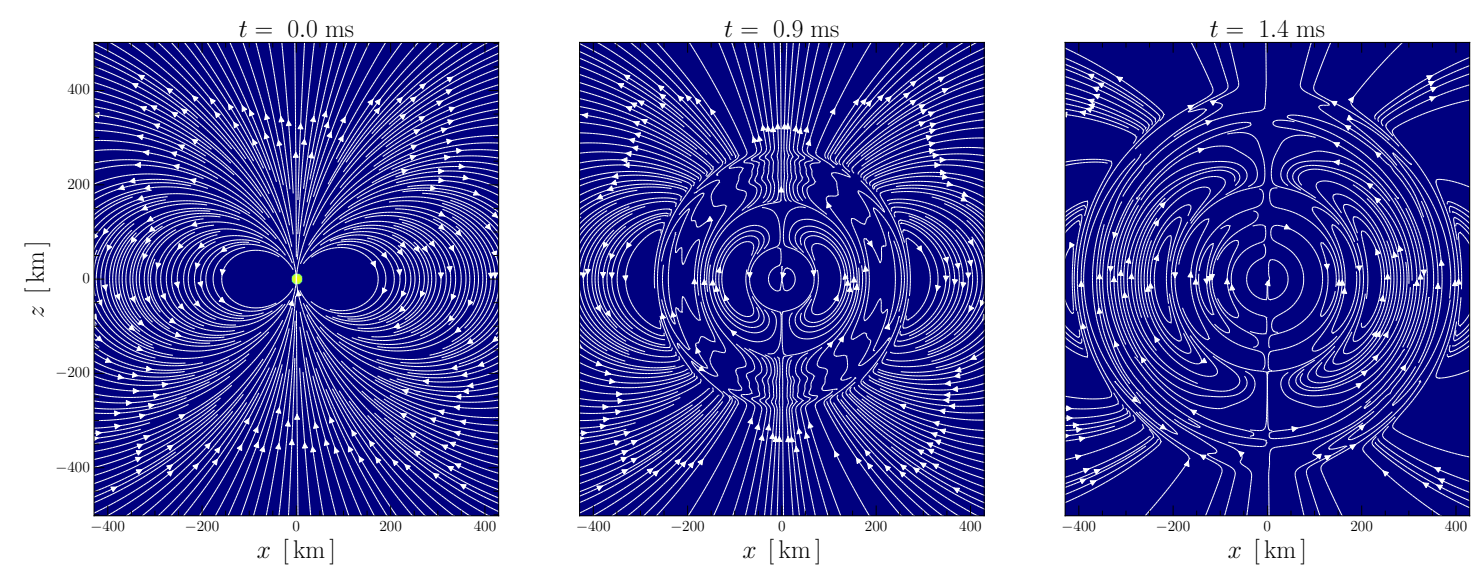

FIG. 2.- Evolution of the magnetic-field lines in the $(x, z)$ plane for the same initial model F000 . B 13 shown in Fig. 1, but presented here on a larger scale to highlight the global structure of the propagating EM wave. The initial neutron star is indicated in green in the left panel as reference scale and the apparent horizon is not included in the middle and left panel, as it is too small for the scales considered.

starts to ringdown, magnetic loops are generated right outside the horizon and then propagate outwards. At this point in time, no magnetic-field lines passes through the horizon and the magnetic-field lines strength has decreased considerably.

Although what described above refers to a nonrotating model, the overall magnetic-field evolution is quite similar, at least on large scales, also for the rotating ones. This is shown in Fig. 3, which is the same as in Fig. 1, but refers in the top row to the initial model $\mathrm{F} 300$. B13, i.e., a neutron star rotating at $300 \mathrm{~Hz}$ and with a pole magnetic field of $10^{13} \mathrm{G}$, while in the bottom row it refers to the initial model $\mathrm{F} 600$. $\mathrm{B} 13^{6}$. Also in these cases, in fact, as the collapse begins and the neutronstar's surface starts to shrink, the magnetosphere is disrupted and a magnetic shock is produced by the snapped magnetic field lines. Again, a quadrupolar EM radiation is produced near the black hole, which propagates outwards as a travelling EM wave. Note that in the case of rotating collapsing stars, all magnetic loops that are formed near the apparent horizon ac-

${ }^{6}$ We are considering very high spin rates in order to explore the variation of the released EM energy as a function of the stellar rotation. However, typical values for the spin frequency in the blitzar scenario are of a few $\mathrm{Hz}$ only. 

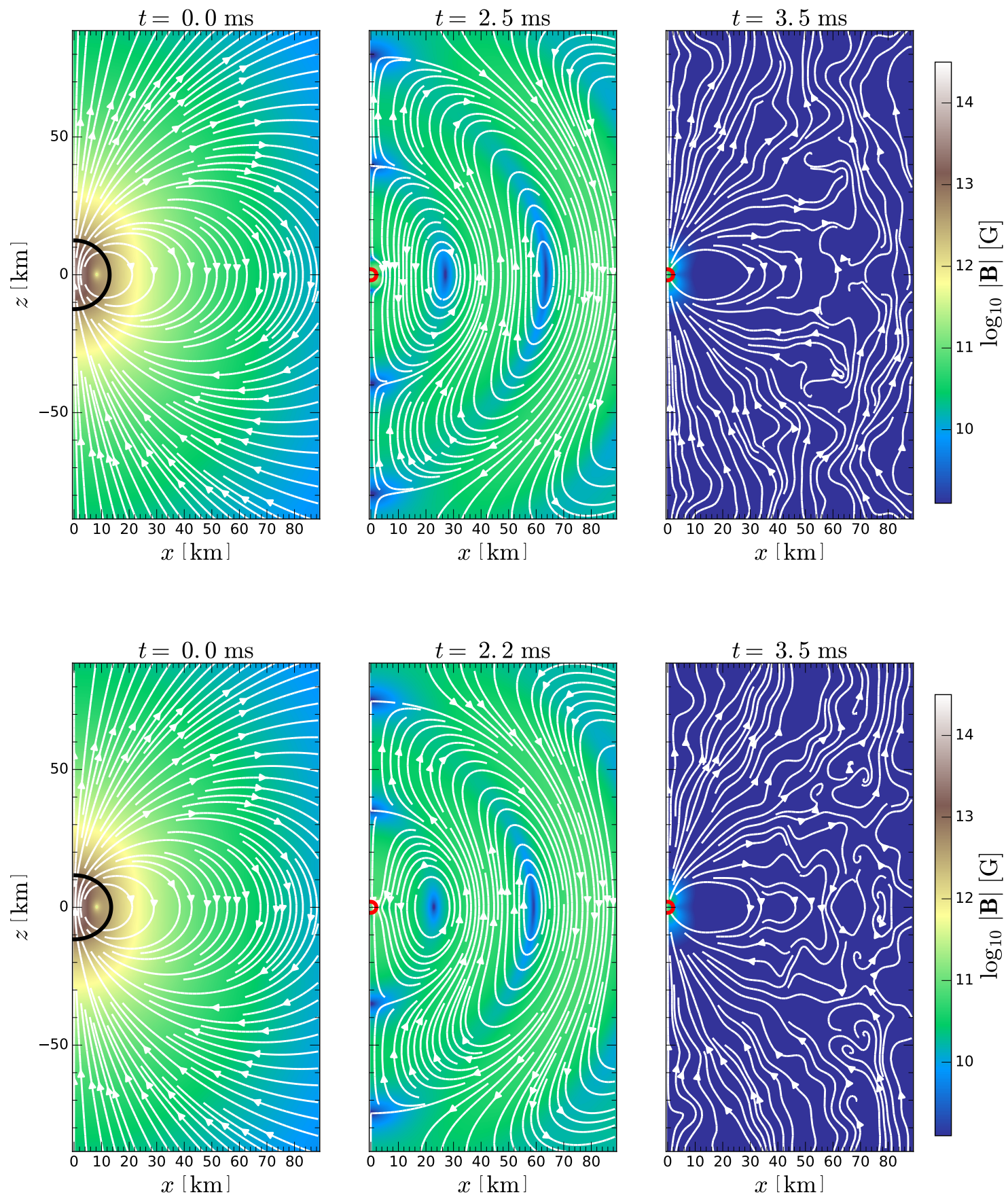

FIG. 3.- Top row: The same as in Fig. 1, but for the case of the initial model F 300 . B13, i.e., a neutron star rotating at $300 \mathrm{~Hz}$ and with a magnetic field of $10^{13} \mathrm{G}$. Note the presence at late times (right panel) of an ordered magnetic since the black hole produced is of Kerr-Newman type. Bottom row: The same as in Fig. 1, but for the case of the initial model F 600 . B13. 

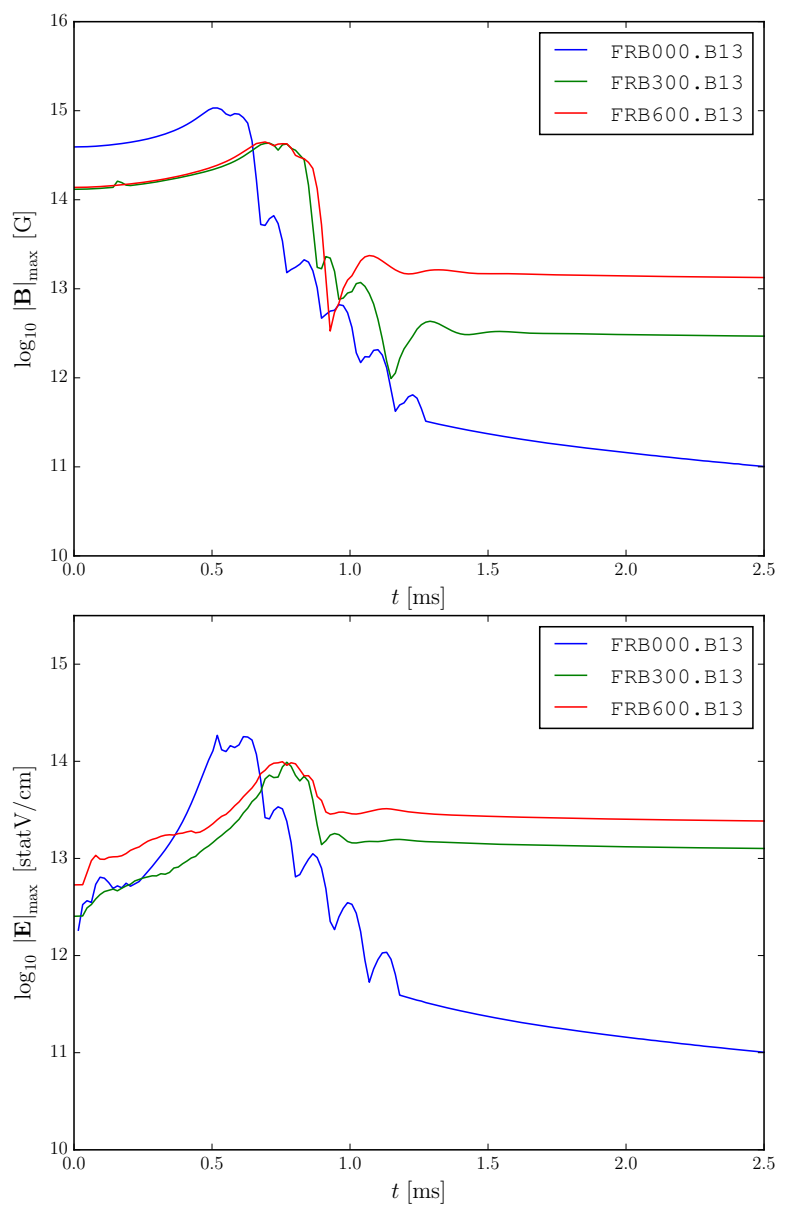

FIG. 4.- Maximum magnetic (left panel) and electric-field (right panel) strengths for selected nonrotating and rotating neutron stars models: F000.B13, F300.B13, and F600.B13.

tually pass through it and are sourced by some current below the apparent horizon.

Another important difference between the rotating and nonrotating models is the different late-time magnetic-field dynamics close to the black hole. This can be appreciated by comparing the right panel of Fig. 1 with the corresponding right panels of Fig. 3. While in fact in the first case the magnetic field is unstructured at all scales, in the second cases the magnetic field exhibits a clear dipolar structure whose strength depends on the initial rotation of the collapsing star: a higher rotation rate yields a higher asymptotic magnetic field at the horizon. This is shown in the left panel of Fig. 4, which reports the maximum magnetic-field strengths for the selected nonrotating and rotating neutron stars models: F000.B13, F300.B13, and F600.B13.

The reason behind this different behaviour is simple and has to be found in the fact that the rotating models have an initial charge induced by the nonzero electric field. Indeed, although our prescription for the electric field, i.e., a corotating interior electric field matched to a divergence-free electric field produced by a rotating magnetised sphere, is the one that minimises the induced charge, our rotating neutron star models are electrically charged initially. As a result, their gravitational collapse will not lead to Kerr black holes but, rather, to Kerr-Newman black holes (Nathanail et al. 2017).

Since we do not model any additional process that would change the net charge of the system, e.g., via pair creation, the initial charge of the neutron star is essentially all con- served and is acquired by the black hole. Such a charge $Q$ is only a very small fraction of the mass of the black hole $M_{\mathrm{BH}}$, i.e., $Q \sim 10^{-4} M_{\mathrm{BH}}$, even for the highest rotation model (Nathanail et al. 2017), but it leaves the black hole with EM fields that could be astrophysically significant.

The evolution of the electric field for the three representative cases is shown in the right panel of Fig. 4 and has a behaviour that is very similar to that of magnetic field (left panel). However, it should be borne in mind that the net charge measured is effectively very small and at the limit of the numerical accuracy of our simulations (we recall that our highest spatial resolution is $h=0.1 M_{\odot}$ at most). In reality, however, if such a collapse would take place in an astrophysical scenario, then the abundant free charges that accompany astrophysical plasmas would neutralise it very rapidly, yielding therefore a standard Kerr solution.

Dionysopoulou et al. (2013), but also Baumgarte \& Shapiro (2003) and Lehner et al. (2012), computed the late time evolution of these EM fields in terms of the magnetic flux across a given surface and showed that they decay exponentially, following the ringdown of the newly formed nonrotating black hole. This behaviour has been reproduced by our simulations and can be seen in Fig. 4, both for the magnetic (left panel) and for the electric field (right panel). Hence, and as remarked by Falcke \& Rezzolla (2014), should the EM emission from an FRB be accompanied by an exponentially decaying EM signal, it would provide unambiguous evidence that a black hole has indeed been produced together with the FRB (see also Fig. 5 and related discussion).

\subsection{Electromagnetic-energy emission}

Having established that the collapse of a magnetised neutron star, be it nonrotating or rotating, leads to a magnetospheric destruction and to the production of an intense emission of EM waves, we will next discuss the energetics and the typical duration of these signals so as to compare the results of our simulations with the phenomenology associated with FRBs.

In particular, we compute the EM luminosity generated during the collapse through the expression

$$
L_{\mathrm{EM}}:=\oint_{\Sigma} \boldsymbol{S}_{\mathrm{EM}} \cdot d \boldsymbol{\Sigma},
$$

on a spherical coordinate surface $\Sigma$ at a radial distance $r \simeq$ $205 \mathrm{~km}$ from the collapsing neutron star, where $\boldsymbol{S}_{\mathrm{EM}}$ is the Poynting vector.

Figure 5 reports the computed luminosity (7) as a function of time for three representative models in a linear (left panel) and in a logarithmic scale (right panel), respectively. The signals from the different stellar models are expressed in retarded time and are aligned so that they coincide when the largest peak reaches the detector. Clearly, all of the luminosity curves show a well defined and dominating sub-millisecond pulse, in close analogy with the observations of FRBs (Rane \& Lorimer 2017). Furthermore, the main pulse is always accompanied by both a precursor that is about $10 \%$ smaller and then by a successive pulse that is of similar amplitude (cf. left panel of Fig. 5). Interestingly, this pattern of peaks is rather similar to the one observed for FRB 121002 (Champion et al. 2016), thus highlighting that a blitzar model can accommodate rather naturally the multi peaked phenomenology of FRBs. Furthermore, and as discussed earlier, even when the black hole is formed, the EM emission does not cease and the black hole 

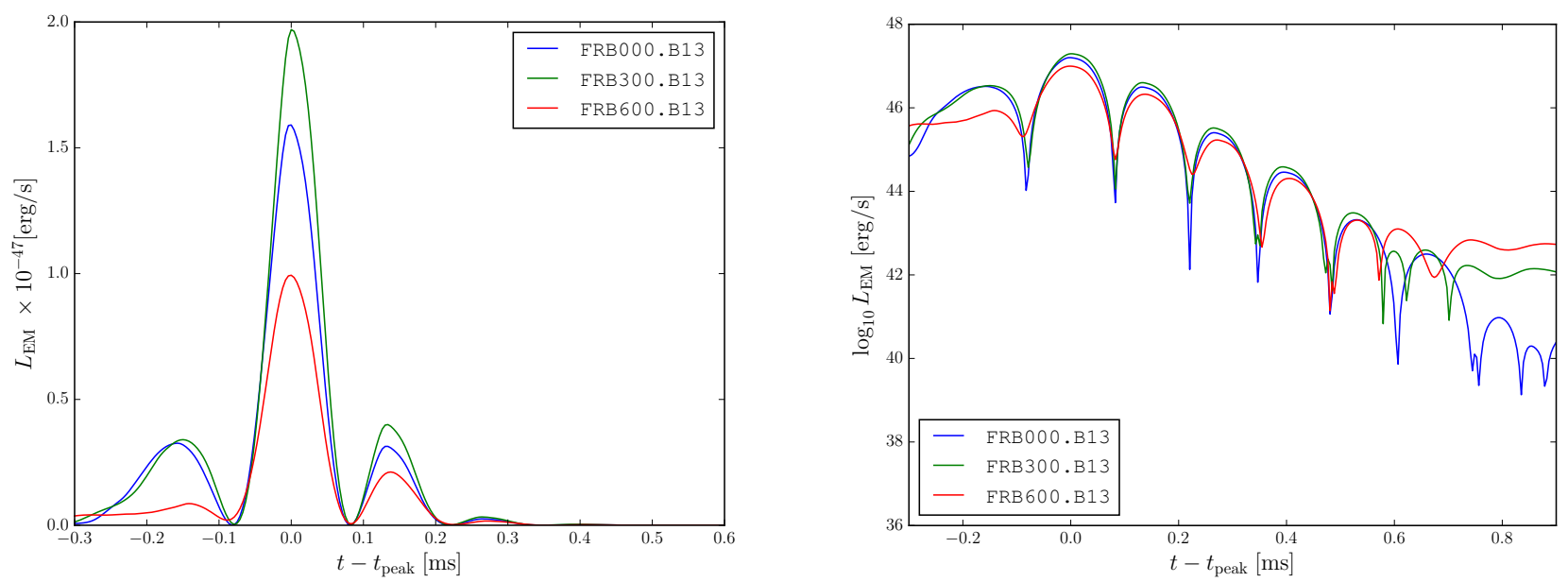

FIG. 5.- EM luminosity for three representative models F000.B13, F300.B13, and F $600 . \mathrm{B} 13$ as extracted at $205 \mathrm{~km}$. The different signals in retarded time are aligned so that they coincide when the largest peak reaches the detector. The two panels report the same data but show it either on a linear scale (left panel) or in a logarithmic one. Note that the small differences in the initial magnetic field strengths at the poles, see Tab. 1, have been scaled out assuming a $B^{2}$ scaling.
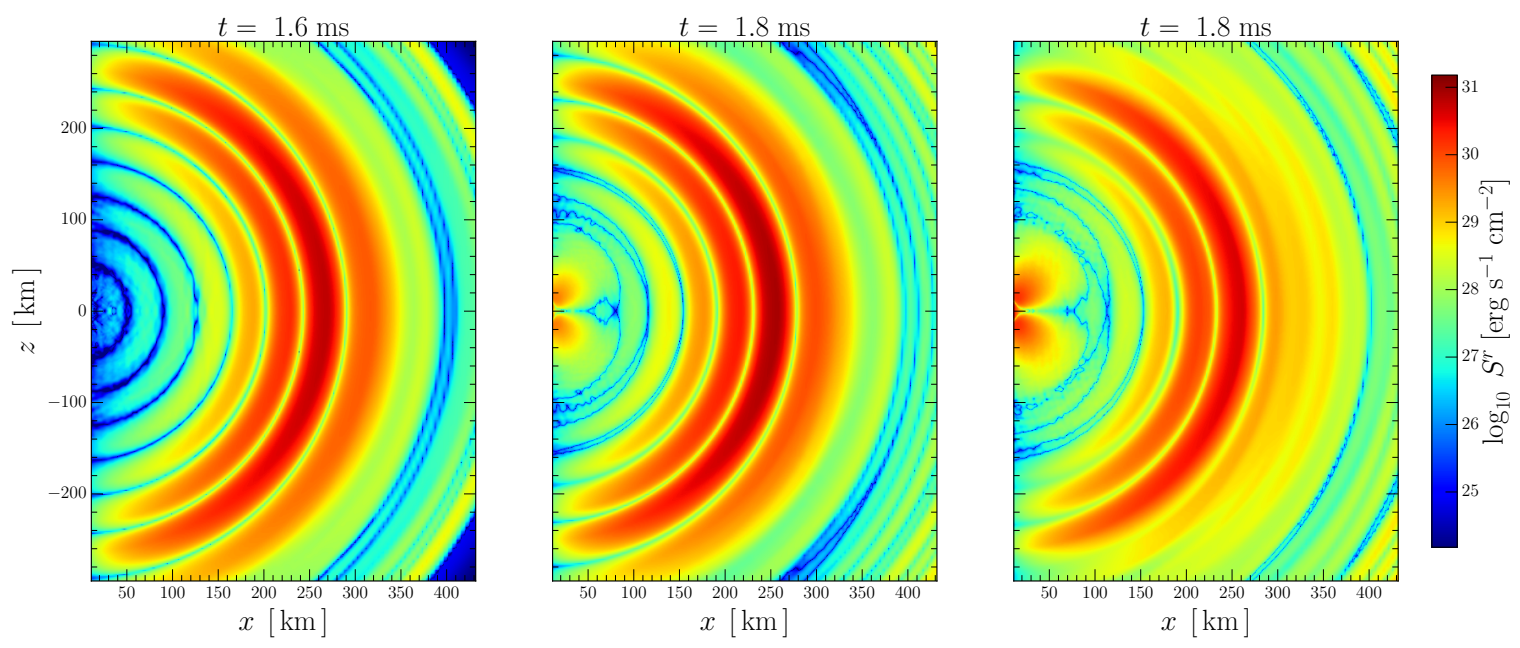

FIG. 6.- Radial component of the Poynting vector $\boldsymbol{S}$ in the $(x, z)$ plane for models F 000 . B13, F 300.B13, and F 600 . B13. It is this complex structure that leads to the multi-peaked EM emission reported in Fig. 5. Note that the small time difference between the models is a result of the slightly delayed collapse for fast rotating models. Note that the small differences in the initial magnetic field strengths at the poles, see Tab. 1, have been scaled out assuming a $B^{2}$ scaling.

rings down shedding its EM perturbations in terms of a wavetrain of EM pulses (cf. right panel of Fig. 5). It is exactly the detection of this ringing-down signature that would corroborate the blitzar model as the most plausible one to describe non-repeating FRBs.

Figure 5 also allows us to deduce two important results that will be further discussed also in the following. Firstly, the overall EM energy radiated in the whole collapse depends only very weakly on the stellar rotation rate (indeed, the radiated energy differs only of $30 \%$ when going from the nonrotating model to the most rapidly rotating model considered, having scaled out the small differences in the initial magnetic field assuming a $B^{2}$ scaling.). Secondly, the timescale for the EM emission is comparable in all cases and $99.99 \%$ of the energy is emitted within one millisecond; this result will be used later on when estimating an expression for the radiated energy.

It is possible to appreciate the multi-peaked structure of the EM emission from the collapsing star through the twodimensional section on the $(x, z)$ plane of the radial component of the Poynting vector $\boldsymbol{S}$ and appears in the integral (7) for the EM luminosity. This is shown in Fig. 6, where all the pulses are visible and distinct as they travel outwards. Considering that the colorcode reports the Poynting vector in a logarithmic scale and that the pulses move at the speed of light, it is to reconstruct from Fig. 6 both the precursor and the exponentially decaying structure of the EM luminosity shown in Fig. 5. Figure 6 also highlights the quadrupolar nature of the EM emission, with most of the intensity concentrated near the equatorial plane of the rotating star. This lack of anisotropy has direct consequences on the event rate of blitzars and detection rate of FRBs, indicating that if blitzars are responsible for FRBs, then the event rate should be close to a factor of two larger than the detection rate. As a final remark, we should point out that the correct event rate of blitzars would be determined by simulating realistic pulsars, meaning that 
the rotational axis is misaligned with the magnetic dipole moment. We intent to extent our present work to the misaligned case.

Having described the overall energetic of the EM emission, it is interesting to correlate the measured radiated energy with the basic properties of the stellar models, namely, the magnetic-field strength and the rotation rate. The ultimate goal is to derive a phenomenological expression that would provide a simple estimate of such quantities on the basis of the measured energetics of the observed FRB. Hence, we compute the radiated energy simply as the time integral of the EM luminosity, i.e.,

$$
E_{\mathrm{EM}}:=\int L_{\mathrm{EM}}(t) d t,
$$

and report in Table 1 the values computed for all of the different models. A rapid look at the table shows that this radiated energy is effectively almost constant across all models and that the radiated EM energy is only weakly dependent on the rate of rotation of the star. This behaviour is rather different from the corresponding energy radiated in GWs $E_{\mathrm{GW}}$. While the two energies are indeed comparable, i.e., $E_{\mathrm{GW}} \approx E_{\mathrm{EM}} \approx 10^{43} \mathrm{erg}$, for a collapsing neutron star with initial magnetic field $B_{\mathrm{pol}} \approx 10^{13} \mathrm{G}$ and rotation frequency $f_{\text {spin }} \approx 100 \mathrm{~Hz}$, the radiated $\mathrm{GW}$ energy has been shown to depend steeply on the dimensionless angular momentum of the star $\tilde{J}:=J / M^{2}$ and, in particular, to follow a relation of the type $E_{\mathrm{GW}} \propto \tilde{J}^{4}$ for rotation rates almost up to the mass-shedding limit (Baiotti et al. 2007). This difference, however, is not surprising and is to be found in the fact that while the EM energy radiated reflects the actual energy stored in the magnetosphere, which does not vary significantly with rotation, the GW energy depends on a high time derivative of the quadrupole moment and is therefore much more sensitive to the variations of the latter with the spin rate.

Next, we take the phenomenological expression proposed by Falcke \& Rezzolla (2014), for the available power in the magnetosphere of a typical pulsar [cf. Eq. (4) of Falcke \& Rezzolla (2014)]

$$
P_{\mathrm{MS}} \simeq 8.4 \times 10^{44} \eta_{\mathrm{B}} t_{\mathrm{ms}}^{-1} b_{12}^{2} r_{10}^{3} \mathrm{erg} \mathrm{s}^{-1},
$$

where $\eta_{\mathrm{B}}$ is the magnetic-energy efficiency, that is, the fraction of magnetic energy in the magnetosphere that is effectively dissipated, $\Delta t=t_{\mathrm{ms}} 1 \mathrm{~ms}$ is the duration of the burst, while $b_{12}$ and $r_{10}$ are the magnetic field of the star and its radius in units of $10^{12} \mathrm{G}$ and $10 \mathrm{~km}$, respectively, i.e., $B_{\text {pol }}=$ : $b_{12} 10^{12} \mathrm{G}$ and $R=: r_{10} 10 \mathrm{~km}$. Note that although $\eta_{\mathrm{B}}$ is unknown (but see below), a value of order unity already provides a value for the luminosity that is in very good agreement with the one observed in FRBs. Here after, we will refer to the magnetic-energy efficiency as $\eta_{\mathrm{Bev}}$, since all our results were obtained assuming an electro-vacuum.

Note that expression (9) assumes a quadratic scaling on the initial magnetic field; while this is reasonable from an energetic point of view, it remains an assumption. However, it can be easily verified by computing the energy emission when considering initial stellar models with the same spin frequency but different degree of magnetisation, i.e., , in terms of the initial models F500.B10-F500.B15. The results of this calculation are shown in Fig. 7, which reports the emitted energy $E_{\mathrm{EM}}$ extracted at $205 \mathrm{~km}$ as a function of the initial value of the magnetic field at the pole $B_{\text {pol. }}$. The log-log plot clearly shows that there is a power scaling between $E_{\mathrm{EM}}$ and

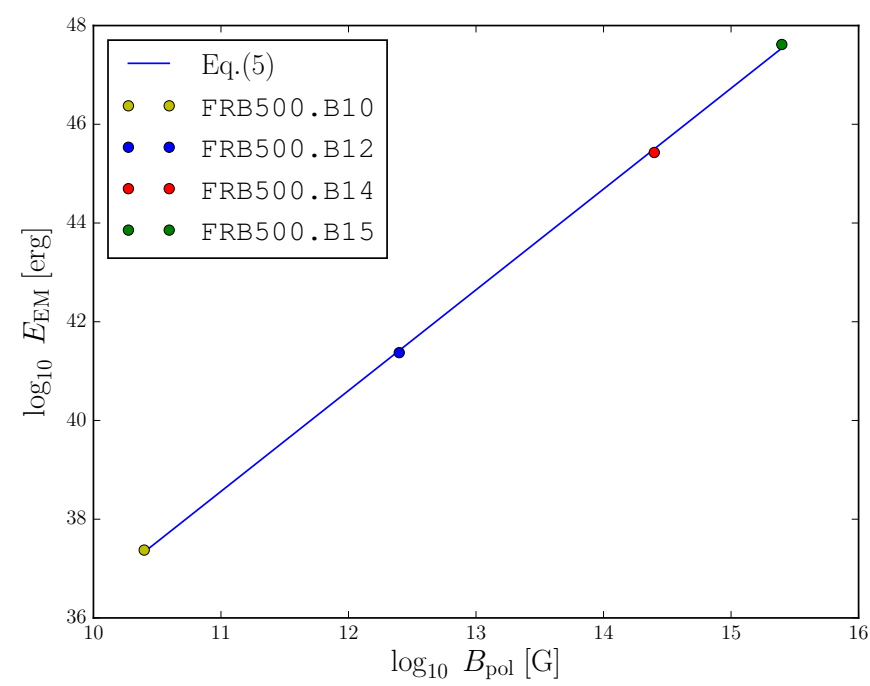

FIG. 7.- Scaling of the emitted EM energy extracted at $205 \mathrm{~km}$ with the initial value of the magnetic field on the pole $B_{\mathrm{pol}}$. The models shown are FRB500.B10-FRB500.B15.

$B_{\text {pol }}$ and a fitting procedure shows that the scaling exponent is indeed 2.04 \pm 0.02 , as predicted by Falcke \& Rezzolla (2014).

The data in Fig. 7 also allows us to fix the magneticenergy efficiency $\eta_{\mathrm{Bev}}$. As mentioned above, in fact, the timescale for the EM emission is essentially independent of the initial stellar rotation rates, at least for the rates considered here (cf. Fig. 5), and is of the order of one millisecond, i.e., $\Delta t_{\mathrm{EM}} / \mathrm{ms}=1=t_{\mathrm{ms}}$. As a result, we can express the emitted energy as

$$
E_{\mathrm{EM}}=P_{\mathrm{MS}} \Delta t_{\mathrm{EM}} \simeq 8.4 \times 10^{41} \eta_{\mathrm{Bev}} b_{12}^{2} r_{10}^{3} \mathrm{erg} .
$$

Using expression (10) and the data in Fig. 7 we therefore deduce via the quadratic fit that the magnetic-energy efficiency is $\eta_{\mathrm{B}}=1.8 \%$ as for the computed models $\mathrm{F} 500$. B10F500.B15. We note that although the efficiency $\eta_{\mathrm{Bev}}$ is only weakly dependent on the initial stellar rotation rate, it is not totally independent of it. Repeating similar calculations also for all stellar models in Table 1 reveals that the higher efficiency of $\eta_{\mathrm{Bev}}=3.6 \%$ is for the model with spinning frequency of $f_{\mathrm{spin}}=100 \mathrm{~Hz}$ and the lowest one $\eta_{\mathrm{Bev}}=1.4 \%$ for the model with spinning frequency of $f_{\text {spin }}=400 \mathrm{~Hz}$. This variance is not unexpected as the dependence of $\eta_{\mathrm{Bev}}$ on $f_{\text {spin }}$ is weak and it is well known that the dynamics of the collapse "slows down" as the spin rate of the neutron stars increases (Baiotti et al. 2007). Given this variance, we compute an average value of $\eta_{\mathrm{Bev}}=(2.1 \pm 0.5) \%$ and hence obtain a phenomenological expression for the EM power released by a blitzar as given by

$$
P_{\mathrm{MS}} \simeq 1.7 \times 10^{43} t_{\mathrm{ms}}^{-1} b_{12}^{2} r_{10}^{3} \mathrm{erg} \mathrm{s}^{-1},
$$

while the corresponding energy is

$$
E_{\mathrm{EM}} \simeq 1.7 \times 10^{40} b_{12}^{2} r_{10}^{3} \mathrm{erg} .
$$

Within the blitzar model, therefore, once an FRB of a given energy is measured, using Eqs. (11) and (12) it is possible, at least in principle, to set constraints on either the radius of the collapsing star or on its magnetic field.

We should remark that the considerations made so far are simply "bolometric" in the sense that we are simply computing the EM energy emitted from the collapsing process in terms of the Poynting flux measured at large distances from 

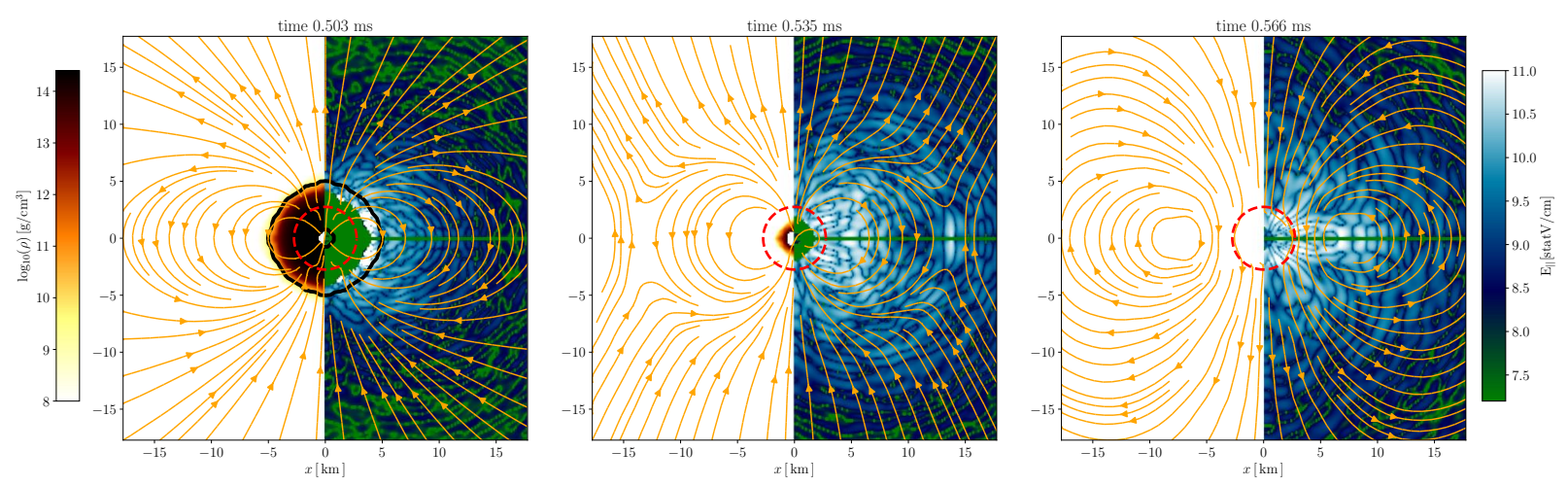

FIG. 8.- Rest-mass density (left pnels) and electric field parallel to the magnetic field (right panels) in the $(x, z)$ plane shown at three different times for model F001.B12. Also reported are the stellar surface (solid black line in the left panel), the apparent horizon (dashed red line), and the magnetic-field lines (orange lines).

the source. In this respect, we have not at all discussed how this bulk energy is then channelled, most likely in a coherent manner, to produce the observed radio emission in FRBs. A simplified curvature-radiation model that uses blitzar emission to reproduce the observed FRB phenomenology is discussed by Falcke \& Rezzolla (2014) and we still consider it a reasonable first radiation model for blitzars. Our future intention is to couple our present simulations with a curvatureradiation model, in order to produce realistic radiation imprints of a blitzar. At the same time, we refer the interested reader to the recent works of Katz (2014); Kumar et al. (2017) and Ghisellini \& Locatelli (2018).

\subsection{On the pair production in a blitzar scenario}

As anticipated in Sec. 3, it is important to assess the occurrence of pair production under the physical conditions that are produced during a blitzar scenario. To this purpose, we follow numerically the evolution of the maximum value of the parallel electric field, $\boldsymbol{E}_{\|}:=\boldsymbol{E} \cdot \boldsymbol{B} /|\boldsymbol{B}|$ responsible for any particle acceleration and hence pair production. Our reference model is that of a typical supramassive neutron star involved in a blitzar scenario, namely, a star with the magnetic field is $B_{\text {pol }}=10^{12} \mathrm{G}$ and period of $1 \mathrm{sec}\left(f_{\text {spin }}=1 \mathrm{~Hz}\right)$ With such initial magnetic field and rotation rate, the star is supposed to have passed its death line, where no pair creation and pulsar emission is expected to take place (Chen \& Ruderman 1993).

In Fig. 8 we show the evolution of the rest-mass density (left portions of the panels) and of the maximum of the parallel electric field (right portions of the panels) at three representative times a typical evolution: one just before all matter is lost inside the black hole and two shortly afterwards; these are also the times when $\boldsymbol{E}_{\|}$reaches its highest value. Also shown in Fig. 8 are the stellar surface (solid black line in the left panel), the apparent horizon (dashed red line), and the magnetic-field lines (orange lines).

Note that as the collapse proceeds, the parallel electric field grows, but also that the largest values are confined within the star and below its surface. Indeed, the parallel electric field remains below the critical value for pair creation $E_{\mathrm{pp}}$ [cf. Eq. (5)]. The maximum of this growth takes place shortly before all matter is lost behind an apparent horizon, so that there is only a very short window in time, i.e., of the order of a fraction of a microsecond, during which charges could be pulled from the stellar surface.

In summary, the results presented in Fig. 8 show that the typical strength of the magnetic field in a blitzar scenario, i.e., $B_{\text {pol }}=10^{12} \mathrm{G}$, is at the limit of the physical conditions below which pair creation is strongly suppressed. This finding provides us with the confidence on the robustness of the results presented here in a pure electrovacuum scenario. On the other hand, the results in Fig. 8 also indicate that for larger initial magnetic-fields, pair creation is very likely to take place. Interestingly, if pair creation does take place during the collapse, the electromagnetic emission is likely be different, hence providing an important signature for the occurrence of the pair creation. More specifically, it is reasonable to assume that together with the emission discussed so far and due to the global snapping of the magnetic-field lines, photons produced from the pair cascade, and that do not have sufficient energy to further pair create, could then diffuse through the stellar exterior leading to an additional emission. This scenario, which could be considered a "dirty blitzar", would then have a multi-frequency radiation spectrum. A more detailed study is necessary to further explore this speculation, both in the theoretical modelling and in the analysis of the observational data.

\section{CONCLUSIONS}

Understanding the physics of astronomical systems dominated by extreme gravity and ultra-strong magnetic fields is at the heart of high-energy astrophysics. In this context, the collapse of a rotating and magnetised neutron star represents a perfect example,. which has been explored via numerical simulations in full general relativity by several authors in the recent past (Baumgarte \& Shapiro 2003; Lehner et al. 2012; Dionysopoulou et al. 2013). In addition to the physical insight that these investigations have brought, they have been also useful to define a theoretical framework that provide a simple explanation of some of the most exciting and yet mysterious astronomical objects that have been recently observed: fast radio bursts. The blitzar model, in fact, involves the gravitational collapse of a rotating and magnetised neutron star and has been proposed early on as a possible and plausible explanation for non-repeating FRBs (Falcke \& Rezzolla 2014). More specifically, this model suggests that an isolated and magnetised supramassive neutron star, i.e., a neutron star whose mass is the maximum mass for nonrotating configurations, collapses when it has lost sufficient angular momentum via the emission of EM energy via dipolar radiation. When this happens, the rotating star disrupts its magnetosphere and launches a coherent EM emission in the radio band (Falcke \& Rezzolla 2014). 
We have here explored the validity of this model going beyond the numerical modelling presented by Dionysopoulou et al. (2013), who considered the gravitational collapse of a magnetised but nonrotating neutron star within a resistiveMHD framework in general relativity. In particular, we have performed accurate numerical simulations of collapsing neutron stars adopting a framework similar to that of Dionysopoulou et al. (2013), but considering here a large number of rotating neutron-star models that differ either in rotation rate or in the initial magnetisation.

Overall, and as observed also in the case of nonrotating stars, we have found that when rotation is involved the disruption of the magnetosphere still takes place on a dynamical timescale. The EM emission is characterised by a precursor signal, followed by a main emission pulse and then by an exponentially decaying signal, typical of the ringdown of black holes from EM perturbations. All the different peaks in EM wave train have a sub-millisecond separation and thus highlight that the blitzar model can easily accommodated multipeaked FRB signals such as the one for FRB (Champion et al. 2016). Furthermore, should the EM emission from an FRB be accompanied by an exponentially decaying EM signal, it would provide unambiguous evidence that a black hole has indeed been produced together with the FRB.

When considering the EM energy properties of the blitzar emission we have found that this is only very weakly dependent on the initial stellar rotation rate, at least for the rotation rates considered here that go up to spin frequency of the fastest known pulsar, i.e., PSR J1748-2446ad. Similarly, the timescale for the EM emission to have decreased by four orders of magnitude is of the order of one millisecond, in reasonable agreement with the observations of FRBs. Exploiting this property and the results of a number of simulations of stellar models that differ only in the initial magnetic-field strength we have been able to show that the radiated EM energy scales quadratically with the magnetic field and that the collapse is able to release in Poynting flux about $2 \%$ of the EM energy initially stored in the magnetosphere. This magneticenergy efficiency is essentially independent of the initial magnetic field and only very weakly dependent on the rotation rates. This results has therefore allowed us to derive a phenomenological expression for the emitted EM energy so that once an FRB of a given energy is measured, it would in principle be possible to set constraints on either the radius of the collapsing star or on its magnetic field if the emission is in- deed produced by a blitzar.

Before concluding we should stress that while the simulations reported here represent a significant progress in the modelling of non-repeating FRBs as blitzars, they also have a number of limitations that call for additional studies and improvements. First, the results presented here are simply "bolometric" in the sense that we are simply computing the EM energy emitted from the collapsing process in terms of the Poynting flux measured at large distances from the source. No attempt has been made to go beyond the curvature-radiation model of Falcke \& Rezzolla (2014) to discuss how the radiated bulk energy is transformed into the observed radio emission in FRBs. While this is beyond the scope of this paper, it is part of our programme of modelling blitzar emission. Second, we have here considered a simplified equation of state to describe the nuclear matter and a single value for the mass of the collapsing star. It would be of great interest to explore how the results presented here change when the stellar models of different masses and different radii are considered.

Finally, while a resistive-MHD approach is a versatile approach to describe the transition between a highly-conductive neutron-star interior and the electrovacuum that should characterise pulsars, it still represents an approximation that can be further improved by varying the choice for the initial electric field, the prescription for the conductivity profile, and possibly the match to a force-free exterior. All of these options will be explored in our future work.

\section{ACKNOWLEDGEMENTS}

It is a pleasure to thank Heino Falcke for useful discussions and input and to the anonymous referee for the considerations on the dirty-blitzar scenario. Partial support comes from the ERC Synergy Grant "BlackHoleCam" (Grant 610058), from "NewCompStar", COST Action MP1304, from the LOEWE-Program in HIC for FAIR, from the European Union's Horizon 2020 Research and Innovation Programme (Grant 671698) (call FETHPC-1-2014, project ExaHyPE). AN is supported by an Alexander von Humboldt Fellowship. The simulations were performed on SuperMUC at LRZ-Munich, on LOEWE at CSC-Frankfurt and on Hazelhen at HLRS in Stuttgart.

\section{REFERENCES}

Baiotti L., Hawke I., Rezzolla L., 2007, Classical and Quantum Gravity, 24, S187

Baumgarte T. W., Shapiro S. L., 2003, Astrophys. J., 585, 930

Bocquet M., Bonazzola S., Gourgoulhon E., Novak J., 1995, Astron. and Astrophys., 301, 757

Bucciantini N., Del Zanna L., 2013, Mon. Not. R. Astron. Soc., 428, 71

Chabrier G., Saumon D., Potekhin A. Y., 2006, Journal of Physics A

Mathematical General, 39, 4411

Champion D. J., et al., 2016, Mon. Not. R. Astron. Soc., 460, L30

Chen K., Ruderman M., 1993, Astrophys. J., 402, 264

Colella P., Sekora M. D., 2008, Journal of Computational Physics, 227, 7069

Cordes J. M., Wasserman I., 2016, Mon. Not. R. Astron. Soc., 457, 232

Dionysopoulou K., Alic D., Palenzuela C., Rezzolla L., Giacomazzo B., 2013, Phys. Rev. D, 88, 044020

Dionysopoulou K., Alic D., Rezzolla L., 2015, Phys. Rev. D, 92, 084064

Falcke H., Rezzolla L., 2014, Astron. Astrophys., 562, A137

Font J. A., et al., 2002, Phys. Rev. D, 65, 084024

Ghisellini G., Locatelli N., 2018, A\&A, 613, A61

Harding A. K., Lai D., 2006, Reports on Progress in Physics, 69, 2631
Harding A. K., Muslimov A. G., 2001, Astrophys. J., 556, 987

Hessels J. W., Ransom S. M., Stairs I. H., Freire P. C., Kaspi V. M., Camillo F., 2006, Science, 311, 1901

Kashiyama K., Ioka K., Mészáros P., 2013, Astrophys. J.1, 776, L39

Katz J. I., 2014, Phys. Rev. D, 89, 103009

Katz J. I., 2016, Astrophys. J., 826, 226

Kumar P., Lu W., Bhattacharya M., 2017, Mon. Not. R. Astron. Soc., 468, 2726

Lehner L., Palenzuela C., Liebling S. L., Thompson C., Hanna C., 2012, Phys. Rev. D, 86, 104035

Liebling S. L., Palenzuela C., 2016, Phys. Rev. D, 94, 064046

Liebling S. L., Lehner L., Neilsen D., Palenzuela C., 2010, Phys. Rev. D, 81, 124023

Löffler F., et al., 2012, Class. Quantum Grav., 29, 115001

Lyubarsky Y., 2014, Mon. Not. R. Astron. Soc., 442, L9

Lyutikov M., Burzawa L., Popov S. B., 2016, Mon. Not. R. Astron. Soc., 462, 941

Masui K., et al., 2015, Nature, 528, 523 

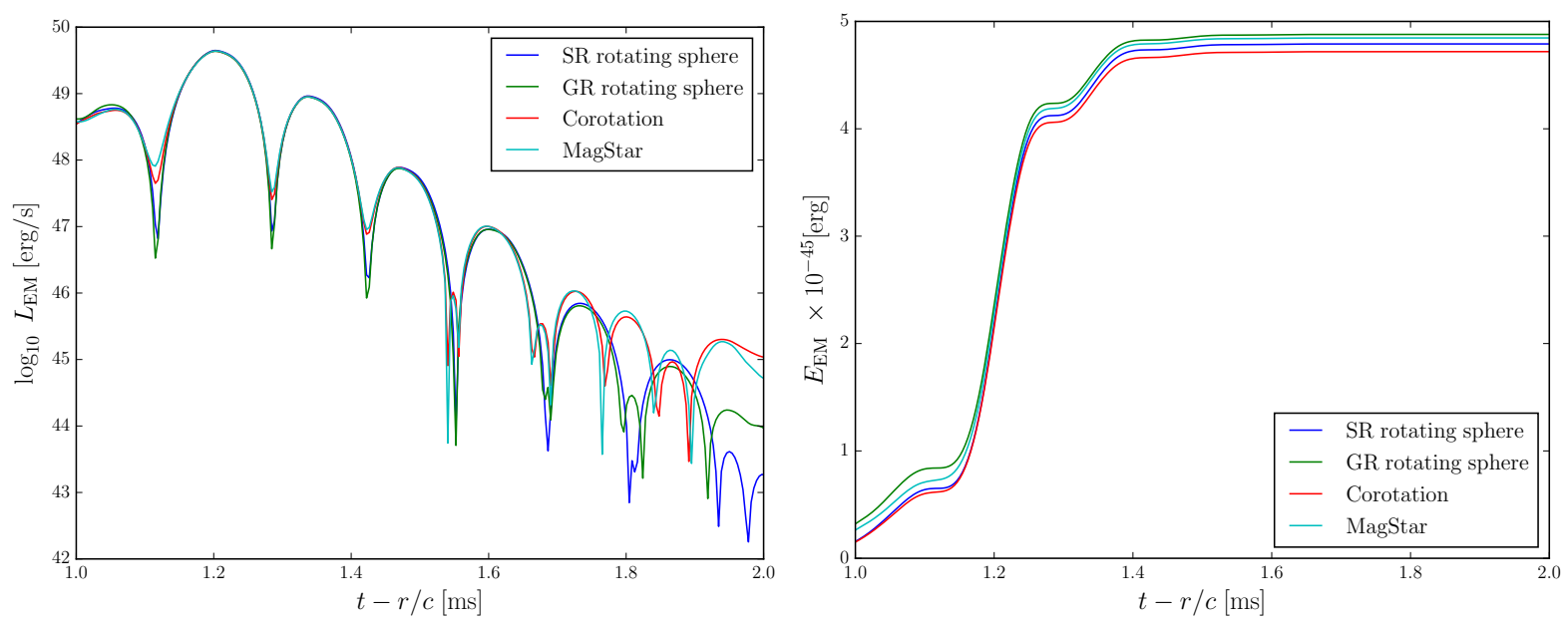

FIG. 9._Left panel: EM luminosity for the different choices of the initial electric field for model F 800 . B13 as extracted at $r \simeq 205 \mathrm{~km}$. Note that the differences among the different prescriptions are effectively very small. Right panel: The same as Fig. 9 but for the emitted EM energy.

Mingarelli C. M. F., Levin J., Lazio T. J. W., 2015, Astrophys. J. Lett., 814, L20

Nathanail A., Most E. R., Rezzolla L., 2017, Mon. Not. R. Astron. Soc., 469, L31

Palenzuela C., 2013, Mon. Not. R. Astron. Soc., 431, 1853

Palenzuela C., Lehner L., Reula O., Rezzolla L., 2009, Mon. Not. R. Astron. Soc., 394, 1727

Pareschi L., Russo G., 2005, Journal of Scientific Computing, 25, 129

Pen U.-L., Connor L., 2015, Astrophys. J., 807, 179

Piro A. L., 2012, Astrophys. J., 755, 80

Popov S. B., Postnov K. A., 2010, in Harutyunian H. A., Mickaelian A. M., Terzian Y., eds, Evolution of Cosmic Objects through their Physical Activity. pp 129-132 (arXiv:0710.2006)

Qian Q., Fendt C., Noble S., Bugli M., 2017, Astrophys. J., 834, 29

Rane A., Lorimer D., 2017, Journal of Astrophysics and Astronomy, 38, 55

Reisswig C., Haas R., Ott C. D., Abdikamalov E., Mösta P., Pollney D., Schnetter E., 2013, Phys. Rev. D, 87, 064023

Rezzolla L., Zanotti O., 2013, Relativistic Hydrodynamics. Oxford University Press, Oxford, UK,

doi:10.1093/acprof:oso/9780198528906.001.0001
Rezzolla L., Ahmedov B. J., Miller J. C., 2001, Mon. Not. R. Astron. Soc., 322,723

Rezzolla L., Ahmedov B. J., Miller J. C., 2003, Mon. Not. R. Astron. Soc., 338,816

Ruderman M. A., Sutherland P. G., 1975, Astrophys. J., 196, 51

Ruffini R., Treves A., 1973, Astrophys. J. Lett., 13, 109

Schnetter E., Hawley S. H., Hawke I., 2004, Class. Quantum Grav., 21, 1465

Sturrock P. A., 1971, Astrophys. J., 164, 529

Takami K., Rezzolla L., Yoshida S., 2011, Mon. Not. R. Astron. Soc., 416, L1

Totani T., 2013, Publications of the ASJ, 65, L12

Wang J.-S., Yang Y.-P., Wu X.-F., Dai Z.-G., Wang F.-Y., 2016, Astrophys. J. Lett., 822, L7

Weih L. R., Most E. R., Rezzolla L., 2018, Mon. Not. R. Astron. Soc., 473, L126

Wilson J. R., 1975, in Bergman P. G., Fenyves E. J., Motz L., eds, Annals of the New York Academy of Sciences Vol. 262, Seventh Texas Symposium on Relativistic Astrophysics. pp 123-132, doi:10.1111/j.1749-6632.1975.tb31425.x

Zhang B., 2016, Astrophys. J. Lett., 827, L31

\section{APPENDIX}

\section{INITIAL ELECTRIC FIELD}

In section 2 we discussed the different possible choices for the initial electric field; in this Appendix we explore the impact they have on the energetic output from a collapsing reference model. To maximise such impact, we consider a rather extreme example, that is, a star rotating at $800 \mathrm{~Hz}$ and with an initial magnetic field of $10^{13} \mathrm{G}$, i.e., model F 800 . B13.

We recall that in view of its infinite conductivity, the electric field for the neutron-star interior is given by the ideal-MHD condition i.e., $E^{i}=-\epsilon^{i j k}\left(v_{\mathrm{c}}\right)_{j} B_{k}$. This interior solution needs to be matched at the stellar surface and then extended to the outer edge of the computational domain in such a way that the closed magnetosphere is corotating and, at the same time, is compatible with our electrovacuum representation of the stellar exterior. In practice, since any choice of an electric field would numerically introduce electric charges, the main goal of the prescription is that of reducing the total net charge and any spurious effect that may arise at the stellar surface

The first choice is to employ the analytic description of the electric field outside a rotating, magnetised and charged sphere in special relativity proposed by Ruffini \& Treves (1973) (we refer to this solution as to "SR rotating sphere"). The second choice is the general-relativistic equivalent of this solution, namely the electric field coming from the analytical description of a rotating magnetised sphere in general relativity (Rezzolla et al. 2001, 2003); this solution is further modified with the addition of monopolar and quadrupolar terms in order to account also for the net electric charge of the star (Ruffini \& Treves 1973). In this way, the modified solution of Rezzolla et al. (2001) is the one that minimises the exterior charge density and is the one that was used throughout the paper (we refer to this solution as to "SR rotating sphere"). The third and fourth choices that we have considered are given respectively by a corotation solution also for the stellar exterior (we refer to this solution as to "Corotation") and by the default solution provided by the Magstar code (we refer to this solution as to "MagStar").

The left panel of Fig. 9 offers a comparison of these four prescriptions for the electric field in terms of the EM luminosity ${ }^{7}$. Clearly, the main features of the luminosity produced during the collapse are all very similar, with some differences becoming

${ }^{7}$ Note that because of its higher rotation rate and magnetic field, model F 800. B13 has a larger luminosity than what shown for the other models 
visible only in the late stages of the evolution, when the luminosity has decreased by about five orders of magnitude and is close to the constant noise level of our code. Similarly, the right panel of Fig. 9 shows a comparison among the total emitted EM energies for the different prescriptions. Also in this case, the different luminosities are very similar and in the range $\sim$ $4.5-5 \times 10^{45} \mathrm{erg}$. Note that the differences reported are actually smaller than the errors introduced when extracting the radiation at different coordinate radii.

In view of the results in Fig. 9, we can safely conclude that the results of our analysis are robust and not influenced by the particular choice of the initial electric field. 